\title{
Aproveitamento do casquilho de soja para a produção de painéis aglomerados convencionais de baixa densidade
}

\author{
Ingrid Luz Guimarães ${ }^{1}$, Maria Cecíllia Ramos de Araújo Veloso², Fernando Jesus Nogara Lisboa ${ }^{3}$, \\ Rafael Farinassi Mendes², Lourival Marin Mendes², Camila Laís Farrapo², José Benedito Guimarães Junior²

\footnotetext{
1 Universidade Federal de Goiás, Campus Samambaia, Goiânia, GO, Brasil. E-mail: ingridg_2507@hotmail.com (ORCID: 0000-0001-8727-7915)

2 Universidade Federal de Lavras, Lavras, MG, Brasil. E-mail: mariacraveloso@outlook.com (ORCID: 0000-0002-8053-180X); rafael_farinassi@hotmail.com (ORCID: 00000002-1530-8388); lourival@dcf.ufla.br (ORCID: 0000-0001-8713-405X); camilafarrapo@hotmail.com (ORCID: 0000-0003-3480-6646); jbguimaraes@hotmail.com (ORCID: 0000-0002-9066-1069)

${ }^{3}$ Universidade Estadual Paulista Júlio de Mesquita Filho, Botucatu, SP, Brasil. E-mail: fernogara@hotmail.com (ORCID: 0000-0003-2949-836X)
}

RESUMO: A madeira é responsável por mais de $40 \%$ do custo de produção de painéis aglomerados e uma das alternativas para atender a demanda da indústria e reduzir o custo de produção desses painéis é o aproveitamento dos resíduos agroindustriais. Nesse sentido, o objetivo deste trabalho foi avaliar o efeito da substituição da madeira por casquilho de soja nas propriedades físicas e mecânicas dos painéis. Os painéis foram produzidos com densidade nominal de $0,60 \mathrm{~g} \mathrm{~cm}^{-3}$. Substituiu as porcentagens de $0,25,50,75$ e 100\% de casca de soja em relação às partículas de eucalipto. As partículas foram encoladas com $12 \%$ do adesivo uréia-formaldeído. $\mathrm{O}$ ciclo de prensagem compreendeu uma pressão de $4,0 \mathrm{MPa}$ e temperatura de $180^{\circ} \mathrm{C}$ durante 15 minutos. Os resultados encontrados mostraram que os painéis aglomerados convencionais produzidos com resíduo de casquilho de soja apresentaram aumento de suas propriedades física e diminuição das mecânicas à medida que se aumentou a substituição da madeira por resíduo. Conclui-se que, do ponto de vista técnico, as propriedades físicas e mecânicas serão atendidas com a substituição máxima da madeira por resíduo de casquilho de soja na proporção de 28,9\%.

\section{Use of soybean hulls for the production of conventional panels of low density agglomerates}

ABSTRACT: Wood accounts for more than $40 \%$ of the production cost of clad of aglomerates panels and to meet industry demand and reduces the production costs of panels, the use of agro-industrial waste is an alternative. In this sense, the objective of this work was to evaluate the effect of the substitution of wood by soybean hull on the physical and mechanical properties of the panels. The panels were produced with a nominal density of $0.60 \mathrm{~g} \mathrm{~cm}^{-3}$. It replaced the percentages of $0,25,50,75$ and $100 \%$ of soybean hulls in relation to the eucalyptus particles. The particles were glued with $12 \%$ of the urea-formaldehyde. The pressing cycle comprised a pressure of $4.0 \mathrm{MPa}$ and a temperature of $180^{\circ} \mathrm{C}$ for 15 minutes. The results showed that the conventional agglomerated panels produced with soybean residue showed an increase in their physical properties and a reduction of the mechanics as the replacement of the wood by residue was increased. It is concluded that, from the technical point of view, the physical and mechanical properties are met with a maximum substitution of the wood per soybean residue in the proportion of $28.9 \%$.

Key words: soybean hulls; bonded plates; Eucalyptus; lignocellulosic material 


\section{Introdução}

No Brasil, as indústrias fabricantes de painéis aglomerados utilizam madeiras provenientes de florestas plantadas (Pierre et al., 2014), principalmente dos gêneros Pinus e Eucalyptus. Para atender à demanda cada vez mais crescente por madeira, há necessidade de não somente aumentar a área de plantios com essas espécies, mas também de diversificar os tipos de matérias-primas utilizadas para a produção dos painéis de partículas (Guimarães Júnior et al., 2011).

Nesse sentido, foi sendo buscado diferentes fontes lignocelulósicas que apresentem potencial para substituírem, em partes ou até mesmo totalmente, a madeira na produção dos painéis particulados. O propósito inicial era aproveitar os resíduos florestais na sua fabricação, porém, essa prática ainda é incipiente em nível industrial, restringindo-se a trabalhos acadêmicos, realizados em escala laboratorial (Guimarães Júnior et al., 2011).

Ainda assim, pesquisadores vêm mostrando a viabilidade do emprego de resíduos agroindustriais de diversas origens para a produção de aglomerados, pois, além de contribuir para o atendimento da demanda de painéis, oferece um destino adequado aos resíduos (Mendes et al., 2010a). Dentre os vários tipos de resíduos lignocelulósicos com potencial para este fim, podem ser citados o sabugo de milho, a casca de café, a casca de amendoim, a casca de soja, o bagaço de cana, entre outros.

O Brasil ocupa a segunda posição em produção mundial de soja em grãos e, segundo levantamento de 2017, obteve safra recorde de $110.161,7$ mil toneladas, sendo a região centrooeste a principal responsável pela safra (CONAB, 2017). Esse grande destaque de produção, consequentemente acarreta em grandes volumes de resíduos. Dessa forma, dentre os resíduos gerados com o processamento da soja estão palha, fibras, cascos e melaço, todos os subprodutos do processo de produção de óleo e proteína da soja (Caillot, 2017).

O casquilho de soja é a película que reveste o grão, e consiste de uma fonte rica em fibra de baixa lignificação (Martins et al., 2015). O descascamento da soja é uma prática comum no processamento de leite de soja, extração do óleo, obtenção de farinhas, isolados e concentrados proteicos, e outros alimentos derivados da soja.

O aproveitamento das cascas de soja normalmente ocorre quando estas são queimadas nas caldeiras, destinadas à geração de calor ou vapor nas próprias indústrias de beneficiamento ou utilizado na alimentação animal devido seu alto teor de fibras. Seguindo a tendência de desenvolvimento sustentável, uma outra alternativa viável é a sua utilização para a produção de painéis aglomerados, já que este produto poderá manter carbono fixo por vários anos, evitando a liberação de gases tóxicos no ambiente após a combustão. Esta alternativa de uso poderá agregar ainda mais valor a este subproduto além de atender à demanda da indústria de painéis por matériaprima, podendo reduzir os custos de produção dos mesmos.

Desse modo, o objetivo deste trabalho foi avaliar o efeito da incorporação da casca do grão de soja nas propriedades físico-mecânicas de painéis aglomerados produzidos com partículas de Eucalyptus grandis.

\section{Material e Métodos}

As cascas de soja foram obtidas em uma empresa que atua no mercado de commodities e operações, na Unidade de Jataí, no sudoeste de Goiás (Figura 1).

A madeira de Eucalyptus grandis foi obtida e processada na Universidade Federal de Goiás, Regional Jataí. Os tratamentos estão descritos na Tabela 1 , sendo produzidos 3 painéis por tratamento, com densidade nominal de $0,60 \mathrm{~g} \mathrm{~cm}^{-3}$.

As partículas "sliver" foram obtidas por meio de moinho de martelo. Estas foram peneiradas, sendo utilizadas para produção do painel aquelas que ficaram retidas entre as peneiras de 10 e 30 mesh. Em seguida, as partículas foram secas até o conteúdo de umidade de $3 \%$ na base seca. 0 adesivo utilizado para o encolamento das mesmas foi a ureiaformaldeído, com teor de sólidos de $64,60 \%$, viscosidade de $480 \mathrm{cP}$, gel time de 51 segundos e $\mathrm{pH}$ de 8,55.

As partículas foram encoladas com $12 \%$ do adesivo, com base no seu teor de sólidos e posteriormente pré-prensadas. O ciclo de prensagem utilizado foi de pressão de 4,0 $\mathrm{MPa}$, temperatura de $180^{\circ} \mathrm{C}$ e tempo de 15 minutos.

Para a avaliação da qualidade dos painéis, foram retirados e avaliados corpos-de-prova de acordo com as recomendações da NBR 14810-3 (ABNT, 2002). Os painéis foram avaliados por meio de suas propriedades físicas de absorção de água e inchamento em espessura após 2 e 24 horas de imersão em água e densidade aparente, sendo retirados 3 corpos-deprova por painel. Para a avaliação da propriedade mecânica de

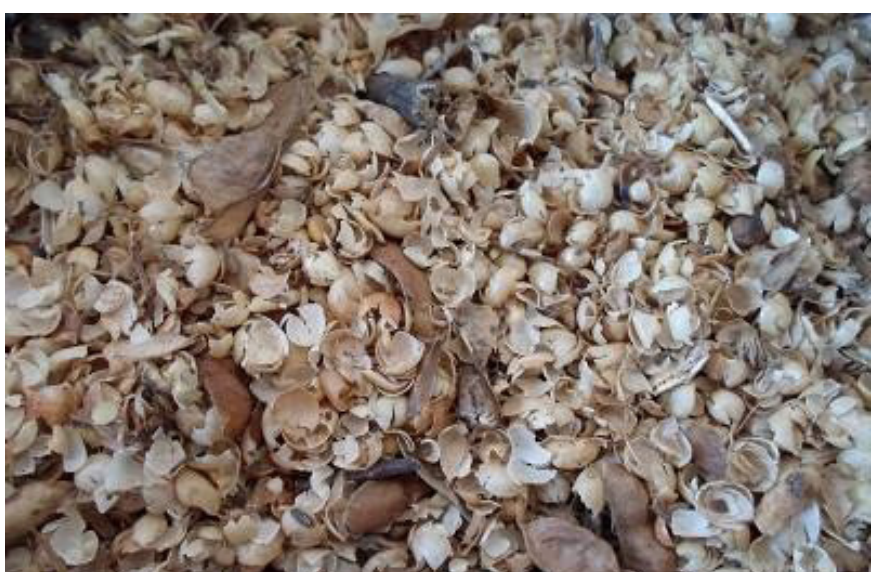

Figura 1. Resíduo de soja - Casquilhos.

Tabela 1. Delineamento experimental utilizado para a produção dos painéis aglomerados.

\begin{tabular}{|c|c|c|}
\hline \multirow{2}{*}{ Tratamento } & Casquilho de soja & Eucalipto \\
\hline & \multicolumn{2}{|c|}{$(\%)$} \\
\hline T1 & 0 & 100 \\
\hline $\mathrm{T} 2$ & 25 & 75 \\
\hline T3 & 50 & 50 \\
\hline T4 & 75 & 25 \\
\hline T5 & 100 & 0 \\
\hline
\end{tabular}


tração perpendicular (TP) foram retirados 4 corpos-de-prova por painel e para flexão estática (com avaliação de módulo de elasticidade/MOE e módulo de ruptura/MOR) amostrou-se 2 corpos-de-prova por painel.

O delineamento experimental utilizado foi do tipo inteiramente casualizado, com 5 tratamentos e 3 repetições. Para as propriedades que apresentaram efeito significativo com a presença da casca de soja foi realizada a análise de regressão linear, a fim de verificar a relação existente entre o aumento da casca de soja e a variação nos valores das propriedades avaliadas dos painéis.

\section{Resultados e Discussões}

\section{Propriedades físicas}

Para a densidade aparente não se observou diferença estatística entre os tratamentos estudados, sendo o valor médio encontrado de $0,584 \mathrm{~g} \mathrm{~cm}^{-3}$ (Figura 2). 0 valor inferior da densidade média dos painéis em relação à densidade nominal de $0,60 \mathrm{~g} \mathrm{~cm}^{-3}$, pode ser atribuída às condições do processo produtivo dos painéis, no qual pode haver perdas de materiais durante o manuseio das partículas nas etapas de aplicação de adesivo, formação do colchão e prensagem dos painéis. Resultados semelhantes foram relatados por diversos autores na literatura (Guimarães Júnior et al., 2011; Mendes et al., 2010b).

De acordo com as normas CS 236-66 (CS, 1968) e ANSI A208.1-99 (ANSI, 1999) todos os painéis produzidos foram classificados como de baixa densidade $\left(<0,60 \mathrm{~g} \mathrm{~cm}^{-3} \mathrm{e}<0,64\right.$ $\mathrm{g} \mathrm{cm}^{-3}$ respectivamente).

Nota-se que houve acréscimo da razão de compactação com o aumento da inserção de resíduo de casquilho de soja no painel, com valores variando de 1,46 a 4,91 (Figura 3). Esse aumento também foi mencionado por outros autores que estudaram o uso de resíduos lignocelulósicos na produção de painéis aglomerados (Silva et al., 2015; Guimarães Junior et al., 2016; Scatolino et al., 2013; Scatolino et al., 2017; Silva et al., 2018), sendo inferido pelos mesmos que a baixa densidade dos resíduos utilizados é a principal responsável pela elevação da razão de compactação.

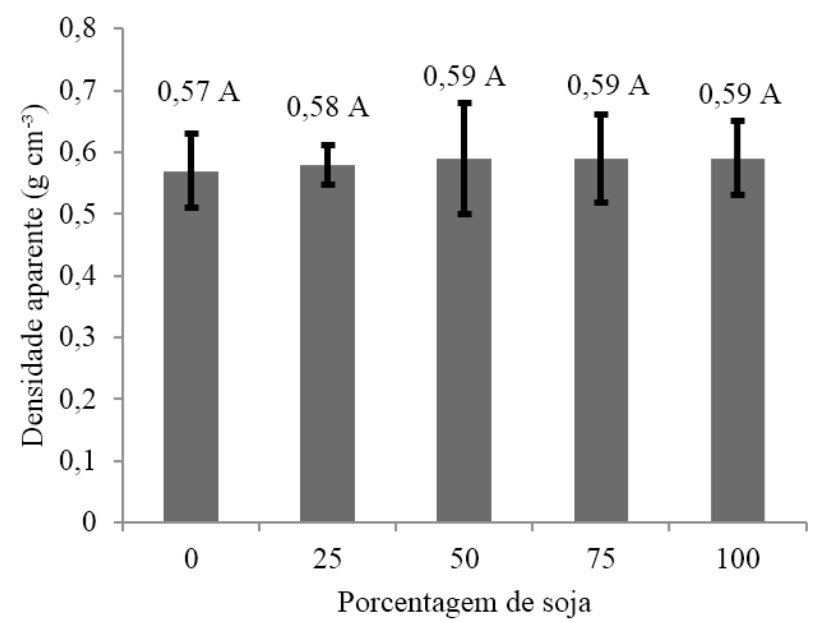

Figura 2. Valores obtidos para densidade aparente dos painéis, de acordo com a porcentagem de casca de soja.

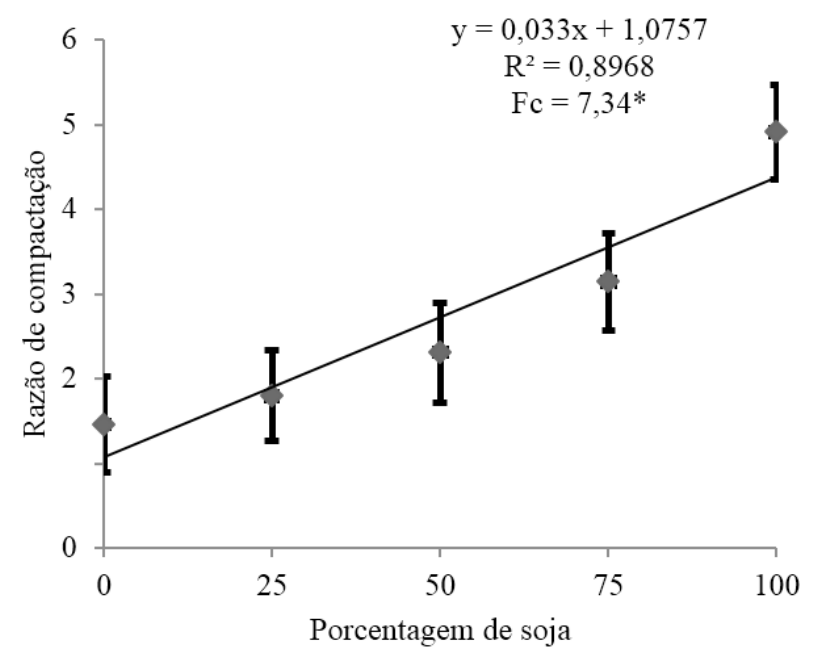

Figura 3. Valores obtidos para razão de compactação dos painéis, de acordo com a porcentagem de casca de soja.

Maloney (1993) relata que a faixa ideal de razão de compactação se encontra entre 1,3 e 1,6. Substituindo esses valores na regressão gerada na Figura 1, observa-se que os valores para atendimento de tal referência se encontram entre 6,8 e $15,9 \%$ de inserção de soja nos painéis aglomerados. Entretanto, vale ressaltar que a faixa de razão de compactação mencionada foi estudada para painéis aglomerados produzidos com madeira e não com resíduos lignocelulósicos. Como estes apresentam como principal característica baixos valores de densidade aparente, já é esperado aumento expressivo de razão de compactação e, talvez, uma nova faixa ideal deva ser estipulada para esse tipo de painel.

Os valores obtidos para a propriedade de absorção de água (AA) em 2 horas de imersão variaram entre 24,9 e 118,59\%, já para 24 horas de imersão, os valores ficaram entre 49,62 e 140,10\% (Figura 4). Em relação ao inchamento em espessura (IE), os valores encontrados variam de 10,00 a $75,95 \%$ e de 17,49 a $80,49 \%$, para 2 e 24 de imersão, respectivamente (Figura 5). Segundo Machado et al. (2017a), as alterações ocorridas nas partículas de madeira e resíduos lignocelulósicos, quando expostos à umidade, tornam-se um fator limitante na utilização da matéria prima.

A tendência de crescimento ocorrida nessas propriedades pode estar relacionada ao aumento da razão de compactação dos painéis. Como aqueles produzidos com maiores quantidades de casquilho apresentaram maior razão de compactação, entende-se que houve um maior número de partículas compactadas em um mesmo volume e,
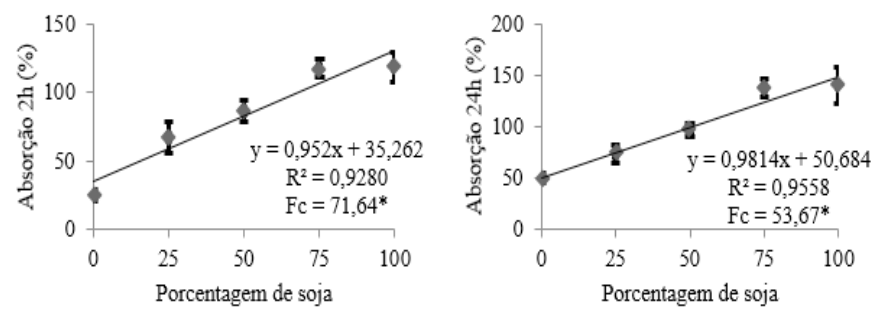

Figura 4. Variação da absorção de água em 2 e 24 horas de imersão, de acordo com a porcentagem de casca de soja. 

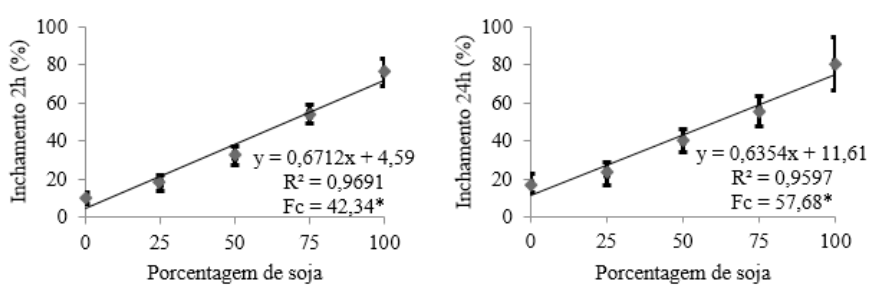

Figura 5. Variação do inchamento em espessura em 2 e 24 horas de imersão em água, de acordo com a porcentagem de casca de soja.

consequentemente, maiores exposições de sítios hidroxílicos podendo levar a uma maior afinidade deste material com a água (Guimarães Junior et al., 2016).

Os valores encontrados neste trabalho para a propriedade de absorção de água foram superiores aos relatados por Mendes et al. (2012), estudando o efeito da associação de bagaço de cana $(25,50$ e $75 \%)$ na produção de painéis aglomerados de Pinus spp., que obteve valores oscilando entre 32,07 e $54,09 \%$ para AA2h e entre 49,82 e $64,17 \%$ para AA24h.

Guimarães et al. (2014), em trabalho realizado com a produção de painéis aglomerados a partir de partículas de pseudocaule de bananeira tratadas quimicamente, encontrou valores similares aos observados neste trabalho, com as propriedades de AA2h, AA24h, IE2h e IE24h variando de 39,8 a $148,8 \%$; de 82,5 a $189,8 \%$; de 9,7 a $55 \%$ e de 15,2 a $74 \%$ respectivamente. Diversos outros autores, estudando substituição da madeira por resíduos lignocelulósicos em painéis, também relatam essa tendência de acréscimo nas propriedades físicas e atribuem esse resultado principalmente ao aumento no número de partículas devido à baixa densidade dos resíduos e à baixa estabilidade dimensional (Barbirato et al., 2014; Machado et al., 2017a; Carvalho et al., 2015)

Os documentos normativos para painéis aglomerados não especificam requisitos para as propriedades de $A A 2 h$ e AA24h. Já para IE24h, a norma de comercialização CS 23666 (CS, 1968) e ANSI A208.1-99 (ANSI, 1999) exigem valores de, no máximo, $35 \%$ para painéis de baixa densidade. Nesse sentido, substituindo esse valor normativo na equação gerada pela regressão, observa-se que seria possível utilizar no máximo de $36,81 \%$ de casquilho de soja para que os valores dessa propriedade atendam às exigências da norma.

\section{Propriedades mecânicas}

Para a tração perpendicular foram encontrados valores médios entre 0,04 e 0,86 $\mathrm{MPa}$, com valores decrescendo de acordo com o aumento da porcentagem de casquilho de soja no painel (Figura 6). Scatolino et al. (2013), incorporando sabugo de milho na produção de painéis aglomerados de Pinus oocarpa, encontrou valores para essa propriedade variando entre 0,25 e 1,08 MPa, os quais foram similares os observados neste trabalho para os painéis produzidos com 0 , 25 e $50 \%$ de casca de soja.

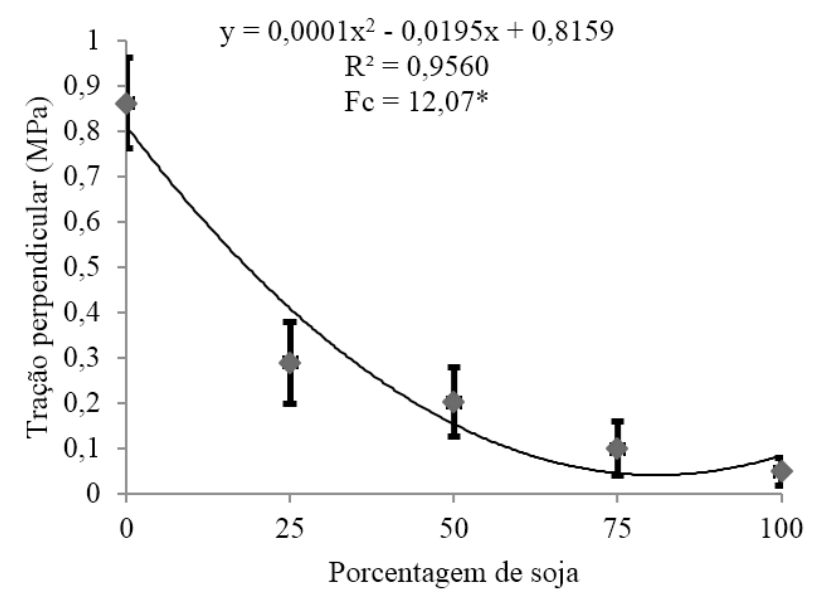

Figura 6. Valores de tração perpendicular - TP em função da porcentagem de casca de soja utilizada.

A norma CS 236-66 (CS, 1968) estabelece valor mínimo de 0,14 MPa para tração perpendicular nos painéis de baixa densidade e produzidos com adesivo ureia-formaldeído para que possam ser comercializados. Dessa forma, observa-se que os painéis produzidos com até $52,4 \%$ de casquilho de soja atenderam à referida norma. Já a ANSI A208.1-99 (ANSI, 1999) preconiza mínimo de 0,10 MPa para essa propriedade fazendo com que até $49,05 \%$ de substituição possa ser feita.

Para as propriedades mecânicas do ensaio de flexão estática (MOR E MOE) notou-se o efeito significativo da substituição da madeira por casquilho de soja nos painéis (Figura 7). Exemplificando esse fato, tem-se que o acréscimo de $1 \%$ de resíduo no painel, promoveu uma redução de, aproximadamente, 0,1071 MPa para o MOR e 15,57 MPa para o MOE.

Cravo et al. (2015) estudando painéis de casca de amendoim verificou que o valor de MOR decresceu quando foi inserido o resíduo. $O$ autor fala que essa propriedade não depende somente da ligação entre as fibras, mas também da força individual da fibra, sua geometria e espessura. Ainda, dizem que as características químicas do resíduo, como teor de celulose e lignina, podem influenciar nas propriedades mecânicas. Neste sentido, pode-se dizer que as características do casquilho de soja precisa ser melhor estudado para melhor entender o motivo da diminuição da resistência.

De acordo com a norma de comercialização CS 236-66 (CS, 1968), os painéis aglomerados de baixa densidade e produzidos
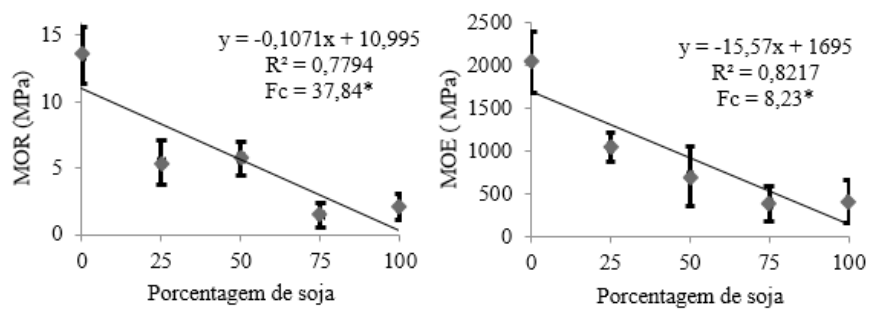

Figura 7. Valores de módulo de ruptura - MOR e módulo de elasticidade - MOE em função da porcentagem de casca de soja utilizada. 
com o adesivo ureia-formaldeído devem apresentar valor mínimo para o MOE de 1029,7 MPa e MOR de 5,5 MPa. Substituindo esses valores de exigência na equação gerada pela regressão, observa-se que o valor máximo de casquilho de soja a ser adicionado ao painel para atendimento da norma é de $42,73 \%$ para MOE e de 51,3\% para MOR. Já para a norma ANSI A208.1-99 (ANSI, 1999) os valores mínimos para MOR e MOE são de $3 \mathrm{MPa}$ e $550 \mathrm{MPa}$ respectivamente. Fazendose a mesma substituição tem-se que a inserção máxima de casquilho que atende a normatização é de $74,65 \%$ para MOR e $71,61 \%$ para MOE.

Machado et al. (2017b) e Fiorelli et al. (2011) em seus estudos com painéis de resíduos de babaçu e cana, respectivamente, observaram que havia decréscimo dos valores de MOR e MOE nos painéis até ficarem abaixo do preconizado pela norma técnica americana (100\% resíduo). Segundo Fiorelli et al. (2011), o aumento na quantidade de matriz garante maior estabilidade dimensional e melhora a adesão entre as partículas, otimizando o desempenho dos painéis.

Esse comportamento de MOE e do MOR em função da adição de resíduos lignocelulósicos nos painéis aglomerados tem sido relatado por diversos outros autores na literatura (Cravo et al., 2015; Scatolino et al., 2013; Guimarães Junior et al., 2016; Scatolino et al., 2017; César et al., 2017; Silva et al., 2018;). Segundo Guimarães Junior et al. (2016) e Scatolino et al. (2017) uma possível explicação para a diminuição das propriedades mecânicas dos painéis aglomerados convencionais consiste na baixa densidade do resíduo e, consequentemente, aumento da razão de compactação, resultando na menor disponibilidade de adesivo por partícula.

\section{Conclusões}

Os painéis aglomerados convencionais produzidos com resíduo de casquilho de soja apresentaram aumento da absorção de água e da instabilidade dimensional e diminuição da resistência mecânica à medida que se foi acrescentado o resíduo.

A quantidade máxima de casquilho que pode ser adicionada, sem prejuízo às propriedades físicas e mecânicas dos painéis, é de $36,81 \%$.

\section{Agradecimentos}

À Fundação de Amparo à Pesquisa de Minas Gerais - FAPEMIG e ao Conselho Nacional de Desenvolvimento Científico e Tecnológico - CNPq que colaboraram para realização desse trabalho.

\section{Literatura Citada}

American National Standards Institute - ANSI. Particleboard standard - ANSI. A208.1-1999. Washington: ANSI, 1999.

Associação Brasileira de Normas Técnicas - ABNT. NBR 14810-1,2,3. Chapas de madeira aglomerada: métodos de ensaio. Rio de Janeiro: ABNT, 2002. 32p.
Barbirato, G.; Fiorelli, J.; Barrero, N. G.; Pallone, E. M. de J. A.; Lahr, F. A. R.; Cristoforo, A. L.; Savastano Júnior, H. Painel aglomerado híbrido de casca de amendoim reforçado com partículas de madeira itaúba. Ciência Florestal, v. 24, n. 3, p. 685-697, 2014. https://doi.org/10.5902/1980509815726.

Caillot, V. A. Avaliação do potencial de produção de biogás dos resíduos da suinocultura codigeridos com resíduos agricultura brasileira. Ponta Grossa: Universidade Tecnológica Federal do Paraná, 2017. 84p. Dissertação Mestrado. http://repositorio. utfpr.edu.br/jspui/handle/1/2386. 19 Jan. 2018.

Carvalho, A. G.; Andrade, B. G. de.; Cabral, C. P. T.; Vital, B. R. Efeito da adição de resíduos de poda da erva-mate em painéis aglomerados. Revista Árvore, v.39, n.1, p.209-214, 2015. https:// doi.org/10.1590/0100-67622015000100020.

César, A. A. S.; Bufalino, L.; Mendes, L. M.; Mesquita, R. G. A.; Protásio, T. P.; Mendes, R. F.; Andrade, L. M. F. Transforming rice husk into a high-added value product: potential for particleboard production. Ciência Florestal, v. 27, n. 1, p. 303-313, 2017. https://doi.org/10.5902/1980509826468.

Commercial Standard - CS. Mat formed wood particle board: CS-23666. Wallingford: CS, 1968.

Companhia Nacional de Abastecimento - CONAB. Acompanhamento da safra brasileira: Grãos - Safra 2016/17 - Sétimo Levantamento, v. 4, n.7, Brasília, Abril 2017. http://www.conab.gov.br/ OlalaCMS/uploads/arquivos/17_04_17_17_20_55_boletim_ graos_abr_2017.pdf. 07 Jan. 2018.

Cravo, J. C. M.; Sartori, D. de L.; Fiorelli, J.; Balieiro, J. C. de C.; Savastano Júnior, H. Painel aglomerado de resíduos agroindustriais. Ciência Florestal, v. 25, n. 3, p. 721-730, jul.-set., 2015. https://doi. org/10.5902/1980509819675.

Fiorelli, J.; Lahar, F. A. R.; Nascimento, M. F.; Sasvastano, H.; Rossignolo, J. A. Painéis de partículas a base de bagaço de cana e resina de mamona - produção e propriedades. Acta Scientiarum. Technology, v. 33, n.4, p. 401-406, 2011. https://doi.org/10.4025/ actascitechnol.v33i4.9615.

Guimarães Júnior, J. B.; Mendes, L. M.; Mendes, R. F.; Mori, F. A. Painéis de madeira aglomerada de resíduos da laminação de diferentes procedências Eucalyptus grandis, Eucalyptus saligna e Eucalyptus cloeziana. Cerne, v.17, n.4, p.443-452, 2011. https:// doi.org/10.1590/S0104-77602011000400002.

Guimarães Junior, J. B.; Xavier, M. M.; Santos, T. S.; Protásio, T. P.; Mendes, R. F.; Mendes, L. M. Inclusão de resíduo da cultura de sorgo em painéis aglomerados de eucalipto. Pesquisa Florestal Brasileira, v. 36, n. 88, p. 435- 442, 2016. https://doi. org/10.4336/2016.pfb.36.88.1036.

Guimarães, B. M. R.; Mendes, L. M.; Tonoli, G. H. D.; Bufalino, L.; Mendes, R. F.; Guimarães Junior, J. B. Chemical treatment of banana tree pseudostem particles aiming the production of particleboards. Ciência e Agrotecnologia, v. 38, n.1, p.43-49, 2014. https://doi.org/10.1590/S1413-70542014000100005.

Machado, N. A. F.; Andrade, H. A. F. de.; Furtado, M. B.; ParraSerrano, L. J.; Parente, M. de O. M.; Silva-Matos, R. R. S. da S. Propriedades físico-mecânicas de painéis multicamadas produzidos com partículas de coco babaçu e de Pinus sp. Revista Agro@mbiente On-line, v. 11, n. 3, p. 191-199, 2017b. https:// doi.org/10.18227/1982-8470ragro.v11i3.4153. 
Machado, N. A. F.; Furtado, M. B.; Parra-Serrano, L. J.; Parente, M. de O. M.; Fiorelli, J.; Savastano Júnior, H. Painéis aglomerados fabricados com resíduos do coco babaçu. Revista Brasileira de Ciências Agrárias, v.12, n.2, p.202-209, 2017a. https://doi. org/10.5039/agraria.v12i2a5434.

Maloney, T.M. Modern particleboard and dryprocess fiberboard manufacturing. 2.ed. São Francisco: M. Freeman, 1993. 689p.

Martins, M. F.; Matos, T. N.; Santos, A. A. R. Dos.; Ascheri, P. R. Extração e caracterização da celulose e síntese de carboximetilcelulose a partir da casca de soja. In: Congresso de Ensino, Pesquisa e Extensão da UEG, 2., 2015, Pirenópolis. Anais... Pirenópilis: UEG, 2015. http://www.anais.ueg.br/index.php/cepe/article/ viewFile/5143/2995. 10 Jan. 2018.

Mendes, R. F.; Mendes, L. M.; Abranches, R. A. S.; Santos, R. C. Dos; Guimarães Junior, J. B. Painéis aglomerados produzidos com bagaço de cana em associação com madeira de eucalipto. Scientia Forestalis, v.38, n.86, p.285-295, 2010a. http://www. ipef.br/publicacoes/scientia/nr86/cap16.pdf. 22 Jan. 2018.

Mendes, R. F.; Mendes, L. M.; Guimarães Júnior, J. B.; Mori, F. A.; César, A. M. Da s. Efeito da incorporação de casca de café nas propriedades físico-mecânicas de painéis aglomerados de Eucalyptus urophylla S.T. Blake. Ciência e Agrotecnologia, v.34, n.3, p.610-617, 2010b. https://doi.org/10.1590/S141370542010000300012 .
Mendes, R. F.; Mendes, L. M.; Guimarães Júnior, J. B.; Santos, R. C. Dos; César, A. A. Da Silva. Efeito da associação de bagaço de cana, do tipo e do teor de adesivo na produção de painéis aglomerados. Ciência Florestal, v. 22, n.1, p.161-170, 2012. https://doi.org/10.5902/198050985088.

Pierre, F. C.; Ballarin, A. W.; Lara Palma, H. Caracterização física de painéis aglomerados de Eucalyptus grandis com adição de resíduos industriais madeireiros. Cerne, v. 20, n. 2, p. 321-328, 2014. https://doi.org/10.1590/01047760.201420021289.

Scatolino, M. V.; Costa, A. O.; Guimarães Junior, J. B.; Protásio, T. P.; Mendes, R. F.; Mendes, L. M. Eucalyptus wood and coffee parchment for particleboard production: physical and mechanical properties. Ciência e Agrotecnologia, v. 41, n. 2, p. 139-146, 2017. https://doi.org/10.1590/1413-70542017412038616.

Scatolino, M. V.; Silva, D. W.; Mendes, R. F.; Mendes, L. M. Use of maize cob for production of particleboard. Ciência e Agrotecnologia, v.37, n.4, p.330 - 337, 2013. https://doi.org/10.1590/S141370542013000400006.

Silva, D. W.; Farrapo, C. L.; Pereira, D.; Mendes, R. F.; Mendes, L. M. MDP com partículas de eucalipto e palha de milho. Scientia Forestalis, v. 43, n. 108, p. 853-862, 2015. https://doi. org/10.18671/scifor.v43n108.10.

Silva, D. W.; Scatolino, M. V.; Prado, N. R. T.; Mendes, R. F.; Mendes, L. M. Addition of different proportions of castor husk and pine wood in particleboards. Waste Biomass Valorization, v. 9, n.1, p. 139-145, 2018. https://doi.org/10.1007/s12649-016-9742-7. 